\title{
A late presentation of an uncommon congenital vascular anomaly
}

\author{
Juvva Kishan Srikanth', Abanti Das², Pranav Ish', Shibdas Chakrabarti', Nitesh Gupta'
}

'Department of Pulmonary Critical Care And Sleep Medicine, Vardhman Mahavir Medical College and Safdarjung Hospital, New Delhi, India

${ }^{2}$ Department of Radiodiagnosis, VMMC and Safdarjung Hospital, New Delhi, India

A 36-year-old male presented with complaints of dyspnea on exertion, cough and sputum for the last 2 years. He had been given anti-tubercular therapy and bronchodilators twice, after a presumptive diagnosis of tuberculosis, but had no relief. He had fresh episode of fever, purulent sputum with scanty hemoptysis and worsened breathlessness for 10 days. There were decreased breath sounds on the left side with the trachea shifted to the left. Sternal percussion had a resonant note. There were no added sounds or bronchial breath sounds. There was no clubbing and the patient had stable vitals. A clinical diagnosis of the left lung collapse was made. The chest X-ray and CT chest were as shown below (Figure 1).
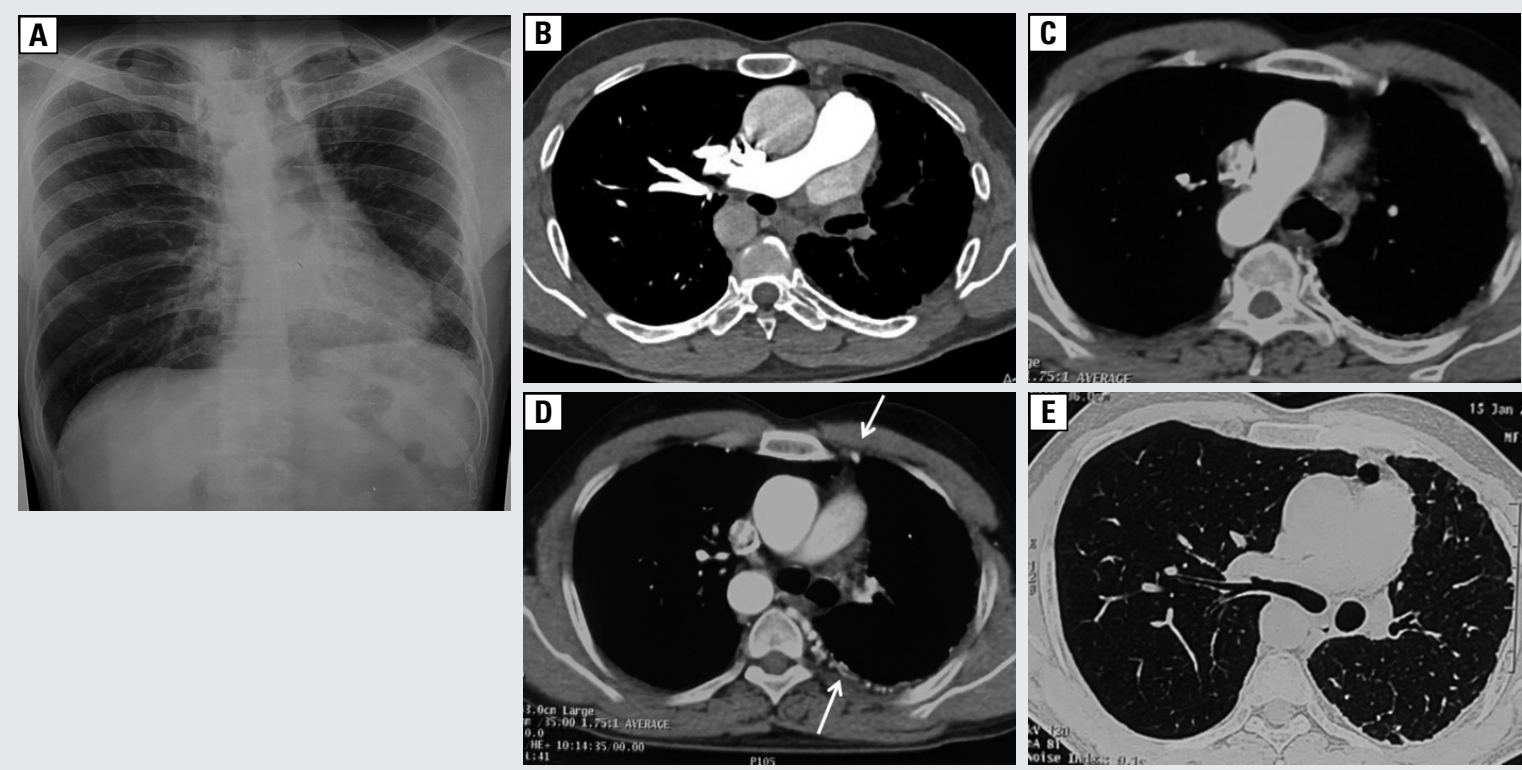

Figure 1. A. Frontal chest radiograph of the patient showing a small volume of the left hemithorax with ipsilateral mediastinal shift, elevation of the left hemidiaphragm and compensatory hyperinflation of the right lung. Diffuse reticular markings are noted peripherally in the left hemithorax with the attenuated left hilum. B. Axial CT angiographic image in pulmonary arterial phase shows the main pulmonary artery leading to the right pulmonary artery with the absence of the left pulmonary arterial stump. C, D. Caudal section in aortic phase shows right-sided aortic arch with hypertrophied internal mammary and intercostal arteries in extra-pleural fat proliferation marked by white arrows. E. Axial lung window shows normal left bronchial development with multiple peripheral reticulations representative of septal collaterals

This is a case of proximal interruption of the pulmonary artery (PIPA) with right-sided aortic arch and compensatory hyperinflation of the right lung.

Most common symptoms are recurrent pulmonary infections, hemoptysis and shortness of breath [1]. Most patients who have no associated cardiac anomalies may have no symptoms and survive into adulthood, which is rare. Most common presentations include exercise intolerance, dyspnea on exertion, hemoptysis or recurrent respiratory tract infections [2].

Address for correspondence: Abanti Das, Department of Radiodiagnosis, Vmmc and Safdarjung Hospital, New Delhi, India; e-mail: das abanti@yahoo.co.in

DOI: 10.5603/ARM.2019.0054

Received: 16.07.2019

Copyright (C) 2019 PTChP

ISSN 2451-4934

Conflict of interest: none declared 
Proximal interruption of the pulmonary artery has a greater incidence on the right side than on the left. The term "interruption" is preferred over "agenesis" due to independent development of intrapulmonary vascular network despite proximal termination at the hilum along with the presence of normal bronchial development, which differentiates this entity from pulmonary agenesis-aplasia-hypoplasia complex [3]. The intrapulmonary vascular network is supplied by hypertrophied systemic collaterals, e.g. bronchial, internal mammary, intercostal and subclavian arteries. Left-sided PIPA is uncommon and generally associated with congenital anomalies like right-sided aortic arch or tetralogy of Fallot, pulmonary atresia, truncus arteriosus and coarctation of the aorta [2]. Our case had an associated right-sided aortic arch. In echocardiography, there were no other congenital anomalies, but there was severe pulmonary artery hypertension (PAH). Such left-sided cases of PIPA often present at a younger age. A series of 108 cases revealed median age of 14 years [4]. Our patient presented at 36 years of age.

Treatment options depend on the age and the symptoms of the patient. Revascularization of the distal pulmonary artery may be done if diagnosed early. Lobectomy or selective embolization of systemic arterial supply can be done for recurrent hemoptysis. It has been reported that $8 \%$ of the patients underwent either a pneumonectomy or a lobectomy for recurrent hemoptysis or intractable pulmonary infections, and $7 \%$ of the patients were subjected to revascularization of hidden pulmonary arteries. It is necessary for these patients to be followed up closely, especially for observation of pulmonary hemodynamics as treatment helps to prevent complications like PAH [4].

Thus, one must keep a high index of suspicion of congenital anomalies of pulmonary vasculature, especially in cases of long-standing respiratory symptoms since childhood, so as to make an early diagnosis, institute appropriate therapy and prevent PAH.

\section{References:}

1. Flores M, Letter H, Derrick E, et al. Unilateral absence of the left pulmonary artery with an associated vascular anomaly in adulthood. Cureus. 2016; 8(3): e527, doi: 10.7759/cureus.527, indexed in Pubmed: 27081588.

2. Kruzliak P, Syamasundar RP, Novak M, et al. Unilateral absence of pulmonary artery: pathophysiology, symptoms, diagnosis and current treatment. Arch Cardiovasc Dis. 2013; 106(8-9): 448-454, doi: 10.1016/j.acvd.2013.05.004, indexed in Pubmed: 23938302.

3. Kieffer SA, Amplatz K, Anderson RC, et al. Proximal interruption of a pulmonary artery. Am J Roentgenol Radium Ther Nucl Med. 1965; 95(3): 592-597, doi: 10.2214/ajr.95.3.592, indexed in Pubmed: 5844925.

4. Ten Harkel AD, Blom NA, Ottenkamp J. Isolated unilateral absence of a pulmonary artery: a case report and review of the literature. Chest. 2002; 122(4): 1471-1477, doi: 10.1378/chest.122.4.1471, indexed in Pubmed: 12377882. 